\title{
Catalysis in flow: Practical and selective aerobic oxidation of alcohols to aldehydes and ketones
}

\author{
Natalia Zotova, ${ }^{a}$ Klaus Hellgardt, ${ }^{b}$ Geoffrey H. Kelsall, ${ }^{b}$ Alan S. Jessiman, ${ }^{c}$ and King Kuok (Mimi) Hii* ${ }^{a}$ \\ Received (in $X X X, X X X) X$ th $X X X X X X X X X 200 X$, Accepted $X$ th $X X X X X X X X X 200 X$ \\ ${ }_{5}$ First published on the web Xth $X X X X X X X X X 200 X$ \\ DOI: 10.1039/b000000x
}

A safe, practical and selective process for the aerobic oxidation of alcohols to aldehydes and ketones has been developed using a Ru catalyst in a continuous flow reactor. Benzylic and allylic alcohols were oxidised selectively to their corresponding aldehydes and ketones, including

10 substrates containing $N$ - and $S$-heteroatoms. Rate of turnover is compatible with that previously reported, using batch or microchannel reactors, under optimised conditions. A preliminary kinetic model was derived, which is supported by experimental observations. Last but not least, tandem oxidation-olefination may be achieved without the need to isolate the alcohol intermediate/solvent switching.

\section{${ }_{15}$ A Introduction}

Development of clean, selective and atom-efficient reactions is the new paradigm in organic synthesis, largely driven by a desire to reduce economic and environmental costs of chemical production. ${ }^{1}$ Arguably, one of the most challenging 20 reactions is the selective oxidation of alcohols to aldehydes and ketones - a process that is ubiquitous in organic chemistry, yet also one of the least efficient, engendering the development of a large number of methodologies. ${ }^{2}$ In recent years, there has been substantial effort devoted to the 25 replacement of stoichiometric oxidants with catalytic methodologies, in an attempt to improve the atom efficiency of the process. ${ }^{3}$

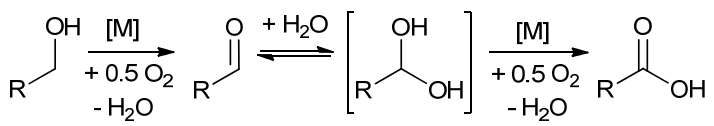

Scheme 1 Oxidation of primary alcohols by $\mathrm{O}_{2}$.

30 One of the most attractive ways of oxidising an alcohol is to use $\mathrm{O}_{2}$ as a terminal oxidant, where only $\mathrm{H}_{2} \mathrm{O}$ is generated as a side-product (Scheme 1). Although there have been a number of heterogeneous catalysts reported to facilitate the process, ${ }^{4}$ there is a genuine concern that the process of mixing 35 the catalyst, reactant and the gaseous oxidant can lead to explosive hazards, particularly if the reaction is performed on a large-scale in a batch reactor. In recent years, the emergence of flow chemistry ${ }^{5}$ provides a solution to the problem, and a number of systems can now be used to conduct these reactions 40 safely. One way of minimising explosive risks is to employ an inert reaction medium, the most popular being supercritical $\mathrm{CO}_{2}$, which has been employed successfully with supported Pd catalysts for the oxidation of alcohol..$^{6-8,9}$ Very recently, the development of a continuous-flow tube reactor for the 45 homogeneous Pd-catalysed aerobic oxidation of alcohols was also reported. ${ }^{10}$ These systems are very effective, but either require specialised equipment to generate and contain supercritical $\mathrm{CO}_{2}$, or require extensive workup (product extraction from reaction media/removal of additives), which 50 may prevent their implementation on a large-scale.

In this paper, we will utilise commercially available catalyst and flow reactor for the selective oxidation of primary and secondary alcohols, delivering a practical synthesis of aldehyde and ketones that fulfils several criteria of green 55 chemistry, exemplified by the following features:

1. Efficient mixing between gas-liquid-solid phases;

2. Good scope and chemoselectivity;

3. Easy to perform: employs commerically available catalyst and flow reactor;

${ }_{60} 4$. Environmentally benign: does not require halogenated solvents, and only water is produced as a side-product;

5. Employs cheap oxidant $\left(\mathrm{O}_{2}\right)$ and heterogeneous catalyst $\left(\mathrm{Ru} / \mathrm{Al}_{2} \mathrm{O}_{3}\right)$. These, and the water side-product, can be easily removed from the product stream, thus affording an easy workup; and

6. Safe: does not involve hazardous reaction conditions or reagents.

\section{B Results and discussion}

\section{Choice of catalyst}

70 There is a plethora of ruthenium catalysts reported for the oxidation of alcohols under aerobic conditions; ranging from monomeric complexes to metal clusters in different oxidation states, supported on a variety of metarials. ${ }^{11-15}$ In this work, $\mathrm{Ru} / \mathrm{Al}_{2} \mathrm{O}_{3}$ was chosen as it is commercially available at a 75 reasonable cost, thus accessible to most synthetic laboratories.

The use of this catalyst for the selective oxidation of alcohols to carbonyl compounds was first proposed by Mizuno et al, ${ }^{16,17}$ where reactions were performed in a batch reactor using trifluorotoluene as a solvent, operating under a 80 constant flow of $\mathrm{O}_{2}$ with azeotropic removal of water. The active catalyst species is believed to be ruthenium hydroxide (Scheme 2), which reacts with the alcohol to generate a ruthenium-alkoxide species that dehydrogenates via a $\beta-\mathrm{H}$ elimination process. The insertion of a dioxygen molecule into 85 the resultant $\mathrm{Ru}-\mathrm{H}$ species forms an unstable peroxide 
complex, which eliminates an oxygen atom to complete the catalytic cycle. ${ }^{17}$ Thus, the overall process consumes an oxygen atom and produces a water molecule as a by-product.

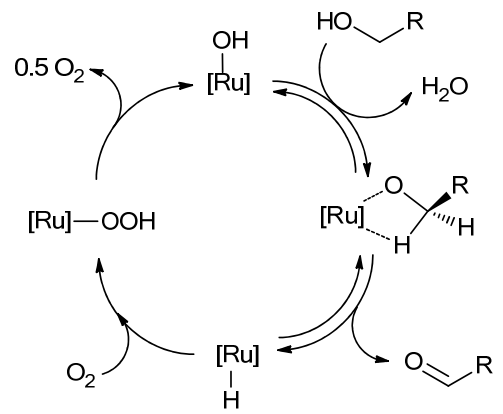

5 Scheme 2 Proposed catalytic cycle for Ru-catalysed oxidation of alcohol.

\section{Description of flow reactor}

$\mathrm{Ru} / \mathrm{Al}_{2} \mathrm{O}_{3}$ has been previously employed as a catalyst in a compact multichannel reactor. ${ }^{18}$ The system consisted of five parallel mm-scaled square packed bed channels, each 10 measuring $100 \mathrm{~mm}$ in length (i.e. $500 \mathrm{~mm}$ in total). As the reaction occurred in a two-phase flow along the length of the reactor, staged injection of oxygen was used to maintain a consistent amount of gas along the reaction bed. Although characteristics of the flow system were very well-defined, the 15 generality of the system was not demonstrated, as only benzyl alcohol was examined as the substrate.

In this work, a commercially-available $\mathrm{XCube}^{\mathrm{TM}}$ reactor was employed, which has a smaller reaction volume (Figure 1): ${ }^{19}$ the catalyst is loaded in one or two cylindrical cartridges 20 (each measuring $4 \mathrm{~mm} \times 70 \mathrm{~mm}$ ), which can be heated and pressurised. In a typical experiment, a solution of the alcohol (0.1-1 $\mathrm{M}$ in toluene) was delivered by a piston pump to a gasmixer, where it was pre-mixed and saturated with the gaseous reactant $\left(\mathrm{O}_{2}\right.$ or air) before it was passed through the catalyst 25 bed. A gas bubble detector maintains a 1:19 bubble-to-liquid ratio, thus the system is not subjected to gas-liquid mass transfer resistance, and the small reactor volume also ensures very little, if any, pressure drop. Residence times of up to 180 seconds may be achieved by controlling the flow rate 30 (normally $1 \mathrm{~mL} / \mathrm{min}$ ), and the product stream can then be collected as separate fractions (for single-pass experiments), or, if desired, re-circulated in continuous flow until reaction is complete.

Using such a setup, a highly laminar flow regime operates 35 in the reactor (calculated Reynold's number, $R_{e}=0.5$ ). A liquid diffusitivity of $5.2 \times 10^{-9} \mathrm{~m}^{2} / \mathrm{s}$ and a Peclet number $\left(P e_{f}\right)$ of 4.5 (see Supporting Information) suggest that mixing occurs mainly by molecular dispersion. The intrinsic large surface-to-volume ratio of the reactor should allow for 40 efficient heat transfer, which is particularly important to prevent thermal runaway processes for these exothermic oxidation reactions. The oxidation of benzyl alcohol to benzaldehyde is calculated to be $-187 \mathrm{~kJ} / \mathrm{mol}$. For the flow reactor operating at $1 \mathrm{~mL} / \mathrm{min}$ with a $1 \mathrm{M}$ solution of alcohol 45 the generated heat was calculated to be $3.12 \mathrm{~J} / \mathrm{s}$. For a single pass conversion, this is significantly smaller (ca. $5.11 \times 10^{-2}$ $\mathrm{J} / \mathrm{s}$ ) than the heat needed to evaporate the toluene solvent
$(4.59 \mathrm{~J} / \mathrm{s})$. With an extremely low adiabatic temperature rise $\left(\Delta \mathrm{T}_{\mathrm{ad}}=1.8{ }^{\circ} \mathrm{C}\right)$, the system operates essentially under 50 isothermal conditions. ${ }^{20}$

The small volume of the reactor ensures that even highly exothermic reactions, such as combustion of toluene, can be effectively contained. Under the maximum pressure of 25 bar, the amount of $\mathrm{O}_{2}$ present in the system is only sufficient to ${ }_{55}$ generate $-97.3 \mathrm{~J}$ of heat from this process, causing $\Delta \mathrm{T}_{\mathrm{ad}}=77$ ${ }^{\circ} \mathrm{C}$ and $\Delta \mathrm{P}=7.1$ bars (due to liberation of $\mathrm{CO}_{2}$ ). Thus, the system can be considered as inherently safe for oxidation reactions.

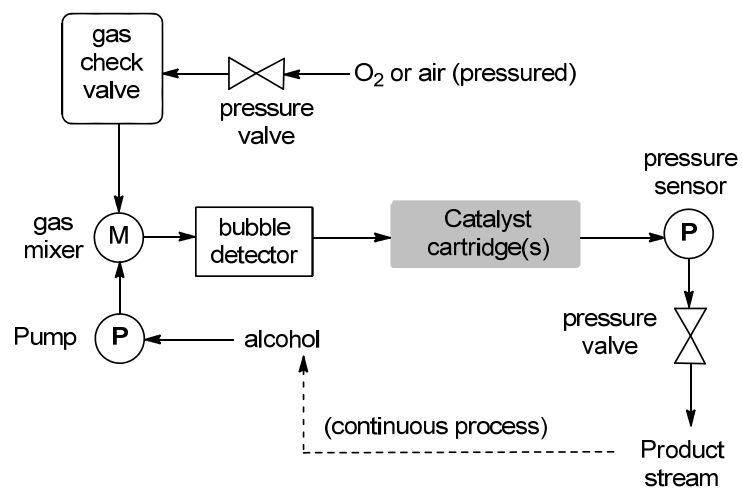

Fig. 1 General schematic of the $\mathrm{XCube}^{\mathrm{TM}}$ flow reactor.

\section{Substrate scope}

A number of primary and secondary alcohols were assessed to establish the scope of the system; the same reaction conditions were applied to provide comparison between the reactivity of 65 the different substrates. The transformation of the benchmark substrate benzyl alcohol to benzaldehyde proceeded smoothly to completion in under an hour with $>99 \%$ selectivity (Table 1, entry 1). Other benzylic alcohols also undergo similar transformation cleanly and selectively, including those 70 containing bulky substituents and/or heteroatoms at the orthoposition (entries 2 and 3). The oxidation of 2-aminobenzyl alcohol, to 2-aminobenzaldehyde is particularly challenging, as the product is prone to self-condense to form imines. In this case, good conversion can be achieved with little side product 75 formation (entry 4).

Most encouragingly, the catalyst remained robust in the presence of pyridyl and thienyl functional groups (entries 47), which are known to inhibit the catalyst in other systems, most notably: $\left[\mathrm{RuCl}_{2}\left(\mathrm{PPh}_{3}\right)_{3}\right] / \mathrm{TEMPO}^{21} \mathrm{Ru}$ deposited on $80 \mathrm{TiO}_{2} / \mathrm{O}_{2},{ }^{22}$ and $\mathrm{Au}$ supported on ceria/air. ${ }^{23}$ In the present system, although a longer reaction time was required for $\mathrm{N}$ containing compounds, the reactions proceeded to completion with $>95 \%$ selectivity.

Allylic alcohols such as cinnamyl alcohol and geraniol can 85 be easily transformed into corresponding conjugated aldehydes (entries 8 and 9). In these cases, the $\mathrm{C}=\mathrm{C}$ moiety remained intact, i.e. no epoxidation/hydrogenation products were detected. Oxidation of secondary alcohols to ketones can also be achieved: 2-phenylethanol was converted to 90 acetophenone $\mathbf{1 0}$ in under an hour (entry 10), while the reaction of aliphatic 2-hexanol to 2-hexanone $\mathbf{1 1}$ was sluggish (entry 11). Conversely, oxidation of the sterically constrained 
cyclooctanol to cyclooctanone 12 was achieved with $64 \%$ conversion (entry 12).

Table 1 Oxidation of alcohols using $\mathrm{Ru} / \mathrm{Al}_{2} \mathrm{O}_{3}$ and $\mathrm{O}_{2}$ under continuous 5 flow. $^{a}$

\begin{tabular}{|c|c|c|c|c|}
\hline Entry & Alcohol & Product $^{b}$ & $\mathrm{t} / \mathrm{h}$ & Conv. ${ }^{c} / \%$ \\
\hline 1 & $\mathrm{PhCH}_{2} \mathrm{OH}$ & $\mathrm{PhCHO}$ & 0.75 & $>99$ \\
\hline 2 & & & 1 & $>99$ \\
\hline 3 & & & 1 & 98 \\
\hline 4 & & & 6 & $94^{d}$ \\
\hline 5 & & & 7 & 95 \\
\hline 6 & & & 7 & 97 \\
\hline 7 & & & 1 & $>99$ \\
\hline 8 & & & 0.75 & $>99$ \\
\hline 9 & & & 2 & $>99$ \\
\hline 10 & & & 1 & $>99$ \\
\hline 11 & $\mathrm{OH}$ & & 7 & $75(91)^{e}$ \\
\hline 12 & & & 7 & 64 \\
\hline 13 & & & 7 & $87^{f}$ \\
\hline 14 & & & 7 & 65 \\
\hline
\end{tabular}

${ }^{a}$ Unless otherwise indicated, reactions were conducted in continuous recirculating mode, where a solution of the alcohol $(0.1 \mathrm{M}, 15 \mathrm{~mL})$ was passed through a cartridge packed with $0.29 \mathrm{~g}$ of $5 \% \mathrm{Ru} / \mathrm{Al}_{2} \mathrm{O}_{3}$ under 5 bars of $\mathrm{O}_{2}$ at $90{ }^{\circ} \mathrm{C}$ and a flow rate of $1 \mathrm{~mL} / \mathrm{min}$ (residence time $=44 \mathrm{~s}$ ).

${ }_{10}{ }^{b}$ Confirmed by ${ }^{1} \mathrm{H}$ NMR spectroscopy or GC, referenced to a genuine sample. Unless otherwise stated, selectivity $>99 \%$. ${ }^{c} \mathrm{GC}$ conversion, using o-xylene as an internal standard. ${ }^{d}<10 \%$ unidentified side-product was observed. ${ }^{e}$ Incorporating a desiccant cartridge. ${ }^{\mathrm{f}}$ Selectivity $=98 \%, 2 \%$ aldol product (vide infra).

15 Aliphatic primary alcohols are challenging substrates for this system. In the batch reactor, the addition of benzoquinone was required to suppress competitive over-oxidation to the acid. Under continuous flow conditions, we were able to attain aldehyde products 13 and 14 from 1-hexanol and 5-hexen-1$20 \mathrm{ol}$, respectively, in moderate conversions, without the need for additives (entries 13 and 14).

Dioxygen was also replaced with zero-grade air (applied at 15 bars) for a few selected reactions (Table 2). No significant difference was observed for conversions of benzyl alcohol and 25 3-thienylmethanol (Table 2, entries 1 and 2 vs Table 1, entries 1 and 7). For slower reactions, such as oxidation of pyridyl alcohols and 1-hexanol (Table 2, entries 3-5 vs Table 1, entries 5, 6 and 12), rates of conversion were noticeably slower, although acceptable yields can still be achieved.

30 Table 2 Oxidation of alcohols using air as oxidant. ${ }^{a}$

$\begin{array}{cccc}\text { Entry } & \text { Alcohol } & \mathrm{t} / \mathrm{h} & \text { Conv./\% } \\ 1 & \text { benzyl alcohol } & 1 & >99 \\ 2 & \text { 3-thienylmethanol } & 1 & >99 \\ 3 & \text { 2-pyridylmethanol } & 7 & 78 \\ 4 & \text { 3-pyridylmethanol } & 7 & 70 \\ 5 & \text { 1-hexanol } & 7 & 80\end{array}$

${ }^{a}$ Reaction conditions are as stated in previous table, except $\mathrm{O}_{2}$ was replaced by air (15 bar). Selectivity for the aldehyde was unchanged in these reactions.

At the end of each reaction, the collected solution was 35 simply evaporated to furnish analytically pure product with $>95 \%$ recovery in all cases. Nine of these product samples were subjected to ICP analysis (Supporting Information), none of which contained detectable level of metal residue $(<0.003 \mathrm{ppm})$. Hence, catalyst leaching is not an issue.

\section{Rate studies}

Next, we examined effects of changing reaction parameters afforded by the reactor. Benzyl alcohol was selected as the model substrate so that our result can be compared to earlier 45 systems.

Assuming that the overall rate is governed by the surface reactions, there are essentially two processes that operate in the catalytic system: (a) the conversion of benzyl alcohol to benzaldehyde with concomitant formation of a water molecule 50 (eqn. 1), and (b) the regeneration of the active catalyst site by dioxygen (eqn. 2).

$$
\begin{gathered}
\mathrm{PhCH}_{2} \mathrm{OH}+[\mathrm{Ru}-\mathrm{OH}] \stackrel{\mathrm{K}_{1}}{\rightleftharpoons} \mathrm{PhCHO}+\mathrm{H}_{2} \mathrm{O}+[\mathrm{Ru}-\mathrm{H}] \\
{[\mathrm{Ru}-\mathrm{H}]+0.5 \mathrm{O}_{2} \stackrel{\mathrm{K}_{2}}{\rightleftharpoons}[\mathrm{Ru}-\mathrm{OH}]}
\end{gathered}
$$

From these, a Mars-van Krevelen-type rate equation can be ${ }_{55}$ derived (eqn. 3 ): ${ }^{24}$

$$
\text { rate }=A \frac{\left[\mathrm{PhCH}_{2} \mathrm{OH}\right]\left[\mathrm{O}_{2}\right]^{0.5}-[\mathrm{PhCHO}]\left[\mathrm{H}_{2} \mathrm{O}\right] / \mathrm{K}_{1} \mathrm{~K}_{2}}{1+\left[\mathrm{O}_{2}\right]^{0.5} \mathrm{~K}_{2}}
$$

where $A=\mathrm{k}_{1} \mathrm{~K}_{2}[\mathrm{cat}]_{\mathrm{tot}}$, [cat $]_{\mathrm{tot}}=$ total catalytic sites.

In the present system (essentially a differential batch recycle reactor), the dissolved $\left[\mathrm{O}_{2}\right]$ is kept constant over the ${ }_{60}$ course of the reaction and that equilibrium is achieved prior to contact with catalyst. Therefore, the rate of the reaction will 
be dependent upon the parameters present in the numerator of eqn. 3 , i.e. it will be expected to be accelerated by increasing the initial concentration of the alcohol, counteracted by the amount of benzaldehyde and water generated during the 5 reaction.

The validity of the equation was tested by following the oxidation of benzyl alcohol at two different initial concentrations ( 0.1 and $0.2 \mathrm{M}$, fig. 2A). Under such dilute conditions, the reaction is pseudo-first order with respect to 10 benzyl alcohol. The rate graphs showed that catalyst activity is reduced at higher concentration, denoting a catalyst deactivation process. By incorporating a desiccant cartridge (containing $1.7 \mathrm{~g}$ of $\mathrm{MgSO}_{4}$ ) into the flow system, the effect can be removed (fig. 2B). Thus, the removal of water 15 accumulated during the reaction has a beneficial effect. This is further illustrated by deploying the desiccant cartridge in the oxidation of 2-hexanol 11 (Table 1, entry 11), where the yield of 2-hexanone can be improved significantly from 75 to $91 \%$.

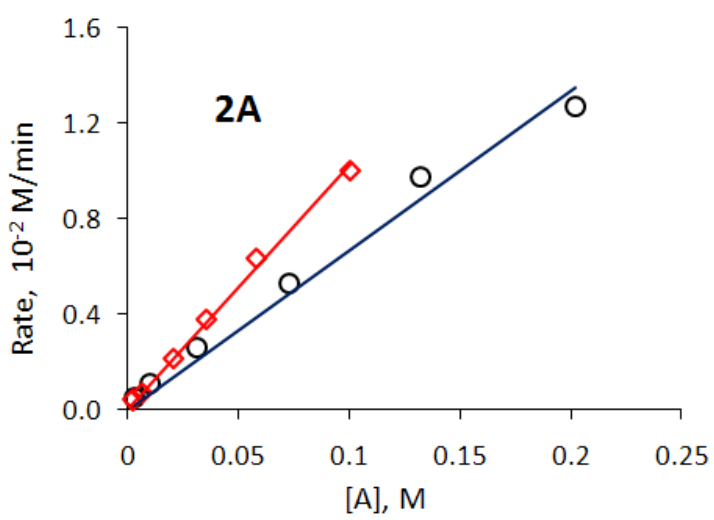

20

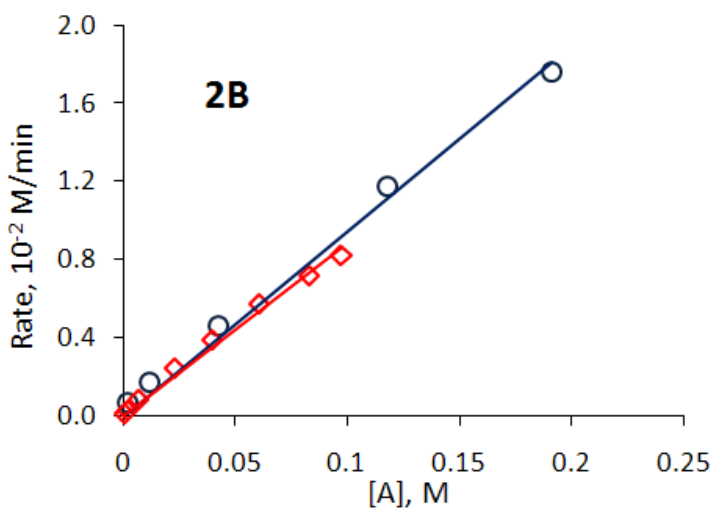

Fig. 2 Rate vs $[\mathrm{A}]$ graphs at two different $[\mathrm{A}]_{0}(\Delta=0.1$ and $\bigcirc=0.2 \mathrm{M})$ in the absence (2A) and presence (2B) of a desiccant cartridge $\left(\mathrm{MgSO}_{4}\right), 5$ bars of $\mathrm{O}_{2},[\mathrm{~A}]=$ [benzyl alcohol].

25 Product inhibition was demonstrated at higher concentrations of alcohol $(0.6$ and $1 \mathrm{M})$. The addition of 0.41 $\mathrm{M}$ of benzaldehyde to $0.6 \mathrm{M}$ of benzyl alcohol caused an overall decrease in its rate of reaction (fig. 3). Notably, the rate profile of the reaction initially emulated that exhibited by 30 the reaction performed with $1 \mathrm{M}$ of the alcohol, but began to deviate beyond $50 \%$ conversion, which is attributed to the difference in the amount of water present in the two systems.

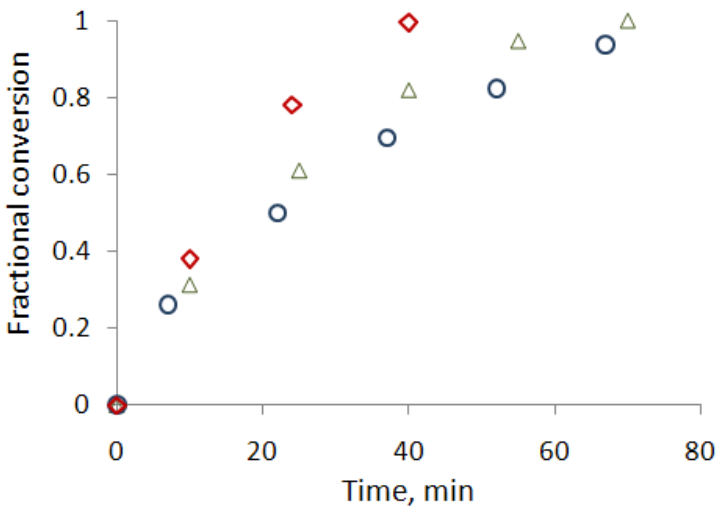

Fig. 3 Conversion graphs with $0.6 \mathrm{M}$ of $[\mathrm{A}]_{0}(\diamond), 1 \mathrm{M}$ of $[\mathrm{A}]_{0}(\mathrm{O})$, and $0.6 \mathrm{M}$ of $[\mathrm{A}]_{0}+0.41 \mathrm{M}$ of benzaldehyde $(\Delta), 5$ bars of $\mathrm{O}_{2}$.

By increasing the $\mathrm{O}_{2}$ pressure from 5 to 25 bars, ${ }^{25}$ the reaction rate was significantly enhanced (fig. 4), restoring the first-order rate of consumption of benzyl alcohol, therefore $\mathrm{O}_{2}$ is integral to the kinetics of the reaction (eqn. 3). This result 40 differs significantly from previous work by Mizuno et al, where $\mathrm{O}_{2}$ was found to have no effect on the rate of reaction (zero order) when it was performed in a batch reactor. ${ }^{17}$ This could be due to the limited range of pressure examined (between 0.2-3 atm), which may not be sufficient for the 45 effect to be discernable experimentally.

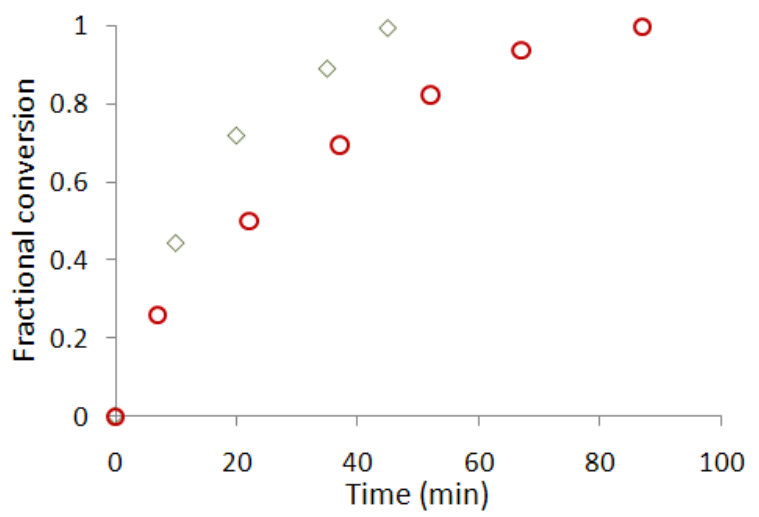

Fig. 4 Effect of increasing $\mathrm{O}_{2}$ pressure. Reaction conditions: $1 \mathrm{M}$ of benzyl alcohol under 5 bars $(\odot)$ and 25 bars $(\diamond)$ of $\mathrm{O}_{2}$.

Although the selectivity of the reactions is $>99 \%$ in most 50 cases, a trace amount of the benzoic acid $(<1 \%)$ can often be detected in the reaction stream by GC, which is known to poison the catalyst. ${ }^{18}$ Indeed, the catalyst loses ca. $20 \%$ of its activity when it was reused in the oxidation of benzyl alcohol, ${ }^{26}$ even though the selectivity of the reaction was 55 maintained. However, catalytic activity can be restored by washing the supported catalyst with an aqueous $\mathrm{NaOH}$ solution. $^{16,18}$

For comparison, the oxidation of 1-hexanol was also performed with and without added product (fig. 5). The 60 conversion was clearly attenuated in the presence of added aldehyde (from $65 \%$ to $c a$. $40 \%$ ), indicating that product inhibition is much more pronounced with this substrate. Furthermore, the formation of the aldol product 15 (Scheme 3) was detected as the reaction progressed by GC (verified by 
independent synthesis), and it is possible that it can also inhibit catalyst activity.
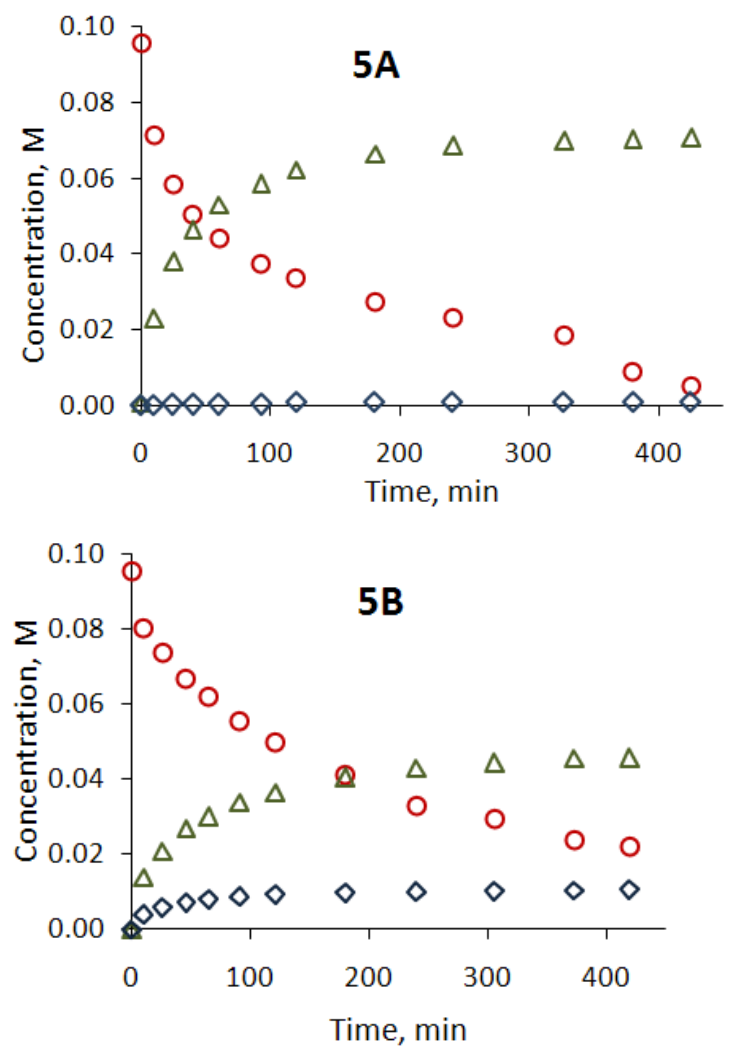

Fig. 5 Reaction profiles for the oxidation of 1-hexanol $(0.1 \mathrm{M})$ under 5 bars of $\mathrm{O}_{2}$ in the absence (5A) and presence (5B) of added hexanal $(0.08$ $\mathrm{M}$, normalised): 1-hexanol $(\circ)$, hexanal $(\Delta)$ and aldol product $(\diamond)$.

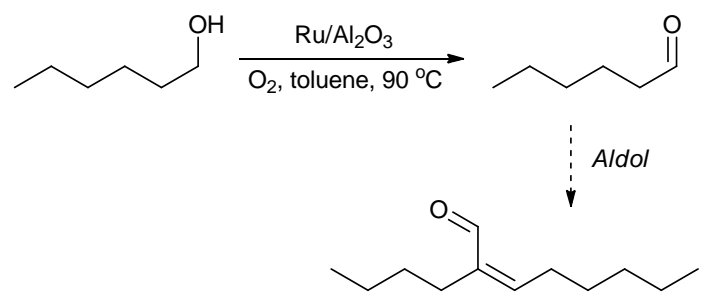

15

Scheme 3 Competitive formation of aldol product.

10 With the limited data in hand, preliminary fitting to eqn. 3 revealed that $\mathrm{K}_{2}$ is approximately two orders of magnitude smaller than $\mathrm{K}_{1}$ for the oxidation of benzyl alcohol, i.e. the formation of benzaldehyde is a much more favourable process than the regeneration of the active catalyst site. This suggests 15 that the catalyst surface is largely populated by $\mathrm{Ru}-\mathrm{H}$ species. Since the regeneration of catalyst is not substrate-dependent, the difference in reactivity between benzyl alcohol and hexanol can be attributed entirely to the difference in $\mathrm{K}_{1}$. This, and other related aspects of the reaction, will be 20 delineated in our future work.

Under these conditions ( $1 \mathrm{M}$ benzyl alcohol and 25 bars of $\mathrm{O}_{2}$ ), 44\% conversion to benzaldehyde can be obtained in 10 min (fig. 4), corresponding to a TOF of $97 \mathrm{~h}^{-1}$. This compares favourably with that reported using the compact multichannel
25 reactor under similar conditions $\left(60 \mathrm{~h}^{-1}\right) .{ }^{18}$ As complete conversion to benzaldehyde can be achieved in $45 \mathrm{~min}$, corresponding to a space-time-yield of $974 \mathrm{~g} \mathrm{~L}^{-1} \mathrm{~h}^{-1}$. This is substantially better than the result obtained with the $\mathrm{Pd}$ catalysed process utilising a sc- $\mathrm{CO}_{2}$ reactor (reported STY of $\left.{ }_{30}>100 \mathrm{~g} \mathrm{~L}^{-1} \mathrm{~h}^{-1}\right)$. $^{7}$

\section{Tandem oxidation-Wittig reactions}

By performing reactions in a flow reactor, the product is obtained as a solution in toluene, which can be isolated simply by evaporating the solvent. However, more commonly, 35 aldehydes and ketones are transformed into further compounds. As a workup procedure is not necessary, this can improve the atom-economy of the process by allowing reactions to be performed in tandem. This was demonstrated by performing oxidation-Wittig reactions in a telescoped 40 synthesis. Previously, a one-pot procedure utilising $\mathrm{Ru} / \mathrm{Al}_{2} \mathrm{O}_{3}$ and a Wittig reagent had been reported for the synthesis of $\alpha, \beta$-unsaturated esters from primary alcohols, ${ }^{27}$ where mixtures of the alcohol, catalyst and Wittig reagent were refluxed in toluene under an $\mathrm{O}_{2}$ atmosphere for 12-24 h, and 45 the final product was purified by column chromatography.

In the present work, methyl (triphenylphosphoranylidene)acetate was added to a solution of cinnamaldehyde collected from the flow reactor. When the olefination was complete, the resultant triphenylphosphine oxide was scavenged using ${ }_{50}$ Merrifield resin. ${ }^{28}$ Following filtration and evaporation, the dienoate 17 can be isolated in $85 \%$ yield, predominantly as the $E, E$-isomer (Scheme 4). The overall procedure, including product isolation, can be achieved in a matter of hours. Crucially, the catalyst can be recovered uncontaminated and ${ }_{55}$ regenerated, if so desired.

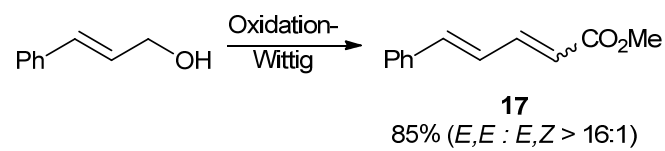

Scheme 4 Telescoped oxidation-Wittig olefination reactions: (i) $\mathrm{Ru} / \mathrm{Al}_{2} \mathrm{O}_{3}, \mathrm{O}_{2}, 90^{\circ} \mathrm{C}, 1 \mathrm{~h}$; (ii) $\mathrm{Ph}_{3} \mathrm{P}=\mathrm{CHCO}_{2} \mathrm{Me}, 3 \mathrm{~h}$.

\section{Conclusions}

${ }_{60}$ A new flow system (differential batch reactor) has been described for the selective oxidation of alcohols to aldehydes and ketones. Reactions were achieved by using commerically available $\mathrm{Ru} / \mathrm{Al}_{2} \mathrm{O}_{3}$ and toluene as a solvent. A variety of primary and secondary alcohols can be converted to their ${ }_{65}$ corresponding carbonyl compounds in good yields and excellent selectivity, with the exception of primary aliphatic alcohols, which gave only moderate conversions. For certain reactions, oxygen can be replaced with air without noticeable decrease in catalyst activity.

70 A global rate equation was derived to model the behaviour of the system. The product and by-product (water) can exert an inhibitory effect on the reaction rate, which can be compensated by increasing the oxygen pressure.

The system can be operated safely even at high pressures of 75 oxygen, and compares very favourably with other flow reactors in terms of turnover rate and space-time-yield. 
Another advantage of the current system is the ease of workup; analytically pure product can be obtained in high yields simply by evaporating the solvent, or used directly in another process without contaminating the catalyst/reagent. 5 This was demonstrated by a telescoped oxidation-Wittig reaction, which was achieved with high yield and selectivity.

\section{Experimental Section}

Starting materials were procured commercially and used without purification. Reactions were performed in reagent-

10 grade toluene. Conversion of alcohol to product was monitored using a HP6890 gas chromatography system fitted with a SPB-5 column $(30 \mathrm{~m} \times 0.2 \mathrm{~mm} \times 0.8 \mu \mathrm{m})$. The percentage of conversion/selectivity was determined by comparing with known standards, using a calibration plot for 15 product and reactant, against o-xylene as an internal standard. the identity of all product was further verified by recording their ${ }^{1} \mathrm{H}$ NMR spectra in $\mathrm{CDCl}_{3}$ using a Bruker Avace NMR spectrometer at $400 \mathrm{MHz}$. ICP analyses (\%Ru) were performed using a Perkin Elmer 2000 DV ICP-OE 20 spectrometer. SEM images were recorded with a JEOL JSM840A microscope, fitted with an Oxford Instruments INCA energy dispersive analytical system (EDS) for elemental X-ray analysis and digital image capture.

Commercially-available $\mathrm{Ru} / \mathrm{Al}_{2} \mathrm{O}_{3}(5 \mathrm{wt} \%)$ was provided by

25 Johnson Matthey plc. BET surface area and mean pore size of the catalyst were obtained by low-temperature $\mathrm{N}_{2}$ adsorption studies at $77 \mathrm{~K}$ using a Micromeritics Tristar 3000 instrument. It was found to have a surface area of $129 \mathrm{~m}^{2} \mathrm{~g}^{-1}$ with a mean pore size of $0.75 \mathrm{~cm}^{3} \mathrm{~g}^{-1}$. The mean pore diameter $=85 \mathrm{~nm}$. ${ }_{30}$ The mean particle size was found to be $100 \mu \mathrm{m}$.

\section{Typical experimental procedure for the oxidation of alcohols.}

Using an $\mathrm{X}-\mathrm{Cube}^{\mathrm{TM}}$, a solution of the alcohol in toluene $(0.1 / 0.2 \mathrm{M}, 15 \mathrm{~mL}$, or $0.6 / 1 \mathrm{M}, 10 \mathrm{~mL})$, containing $\mathrm{o}$-xylene $(0.05 \mathrm{M}$ of $0.5 \mathrm{M})$ as an internal standard, was introduced into 35 a heated cartridge containing 5 wt. $\mathrm{Ru} / \mathrm{Al}_{2} \mathrm{O}_{3}(0.29 \mathrm{~g})$ at 90 ${ }^{\circ} \mathrm{C}$, under 5 or 25 bars of $\mathrm{O}_{2}$. The solution was re-circulated continuously, and the conversion was monitored by GC analysis. Upon completion, the solution was evaporated to afford the corresponding aldehyde/ketone with $>90 \%$ 40 recovery. The purity and identity of the product was verified by ${ }^{1} \mathrm{H}$ NMR spectroscopy.

\section{Procedure for tandem oxidation-Wittig reactions.}

A $0.1 \mathrm{M}$ solution of the aldehyde was generated from the corresponding alcohol using the procedure described above, 45 until complete conversion was indicated by GC. Methyl (triphenylphosphoranylidene)acetate (1.2 eq.) was added in one portion, and stirred at room temperature until all aldehyde has been consumed (TLC, $3 \mathrm{~h}$ ). Merrifield resin ( $1 \mathrm{~g} / 2 \mathrm{mmol}$ of acetate used) and KI (2.5 eq.) were added to the reaction ${ }_{50}$ mixture and stirred for $2 \mathrm{~h}$ at room temperature, to remove any remaining traces of the phosphorus reagent/by product. The insoluble material was removed by filtration through a short pad of silica gel, which was washed repeatedly with $\mathrm{Et}_{2} \mathrm{O}$. The combined filtrate was evaporated to give ${ }_{55}$ analytically pure product $17 .{ }^{1} \mathrm{H}$ NMR spectrum recorded of the residue is given in the Supporting Information.

\section{Acknowledgements}

This work is funded by an EPSRC grant, co-sponsored by Pfizer (EP/G027544/1). We are grateful to Johnson Matthey ${ }_{60} \mathrm{plc}$ for the provision of supported metal catalysts for this project.

\section{Notes and references}

${ }^{a}$ Department of Chemistry, and

${ }^{b}$ Department of Chemical Engineering and Chemical Technology, 65 Imperial College London, Exhibition Road, South Kensington, London SW7 2AZ, U.K. Fax: +44 20 75491142; E-mail: mimi.hii@imperial.ac.uk ${ }^{c}$ Pfizer Global Research and Development, Ramsgate Road, Sandwich CT13 9NJ, U.K.

$70 \dagger$ Electronic Supplementary Information (ESI) available: Description of reactor, calculation of flow characteristics, thermodynamic data, and product analysis, including ICP analysis of metal residues. See DOI: $10.1039 / \mathrm{b} 000000 \mathrm{x} /$

75 1. P. J. Dunn, A. S. Wells and M. T. Williams, eds., Green Chemistry in the Pharmaceutical Industry, Wiley-VCH, Weinheim, 2010.

2. G. Tojo and M. Fernandez, eds., Oxidation of alcohols to aldehydes and ketones: a guide to current common practices, Springer, 2006.

3. R. A. Sheldon, I. W. C. E. Arends, G. J. Ten Brink and A. Dijksman, Acc. Chem. Res, 2002, 35, 774-781.

4. T. Mallat and A. Baiker, Chem. Rev., 2004, 104, 3037-3058.

5. C. Wiles and P. Watts, Eur. J. Org. Chem., 2008, 2008, 1655-1671.

6. G. Jenzer, T. Mallat and A. Baiker, Catal. Lett., 2001, 73, 5-8.

7. Z. Hou, N. Theyssen, A. Brinkmann, K. V. Klementiev, W. Grünert, 85 M. Bühl, W. Schmidt, B. Spliethoff, B. Tesche, C. Weidenthaler and W. Leitner, J. Catal., 2008, 258, 315-323.

8. M. Caravati, J.-D. Grunwaldt and A. Baiker, Appl. Catal., A, 2006, 298, 50-56.

9. Immobilised Pd catalysts have also been employed in a flow reactor 90 in a basic aqueous medium: A. L. Tarasov, L. M. Kustov, A. A. Bogolyubov, A. S. Kiselyov and V. V. Semenov, Appl. Catal., A 2009, 366, 227-231.

10. X. A. Ye, M. D. Johnson, T. N. Diao, M. H. Yates and S. S. Stahl, Green Chem., 2010, 12, 1180-1186.

95 11. K. Yamaguchi, K. Mori, T. Mizugaki, K. Ebitani and K. Kaneda, J. Am. Chem. Soc., 2000, 122, 7144-7145.

12. B. Z. Zhan, M. A. White, T. K. Sham, J. A. Pincock, R. J. Doucet, K. V. R. Rao, K. N. Robertson and T. S. Cameron, J. Am. Chem. Soc., 2003, 125, 2195-2199.

100 13. J. H. Liu, F. Wang, K. P. Sun and X. L. Xu, Adv Synth Catal, 2007, 349, 2439-2444.

14. M. L. Kantam, U. Pal, B. Sreedhar, S. Bhargava, Y. Iwasawa, M. Tada and B. M. Choudary, Adv Synth Catal, 2008, 350, 1225-1229.

15. M. Zahmakiran and S. Orzkar, J. Mater. Chem., 2009, 19, 7112 7118.

16. K. Yamaguchi and N. Mizuno, Angew. Chem. Int. Ed., 2002, 41, 4538-4542.

17. K. Yamaguchi and N. Mizuno, Chem.-Eur. J., 2003, 9, 4353-4361.

18. D. V. Bavykin, A. A. Lapkin, S. T. Kolaczkowski and P. K. Plucinski, Appl. Catal., A, 2005, 288, 175-184.

19. The X-Cube system is available from ThalesNano Nanotechnology Inc, Graphisoft Park, H-1031 Budapest Záhony u. 7, Hungary, see: http://www.thalesnano.com/products/x-cube. Detailed schematic of the system is provided in the Supporting Information.

1520 . Under the relatively mild experimental conditions (0.1-1 $\mathrm{M}$ of alcohol, $90^{\circ} \mathrm{C}, 5-25$ bars of $\mathrm{O}_{2}$ ), the liquid-phase oxidation of toluene via a radical mechanism will be too slow to compete, see: (a) J. A. A. Hoorn, J. Van Soolingen and G. F. Versteeg, Chem. Eng. Res. Des., 2005, 83, 187-195; (b) S. W. Tang and B. Liang, Ind. Eng. Chem. Res., 2007, 46, 6442-6448. 
21. A. Dijksman, A. Marino-Gonzalez, A. M. I. Payeras, I. Arends and R. A. Sheldon, J. Am. Chem. Soc., 2001, 123, 6826-6833.

22. A. Köckritz, M. Sebek, A. Dittmar, J. Radnik, A. Brückner, U. Bentrup, M. M. Pohl, H. Hugl and W. Mägerlein, J. Mol. Catal. A: Chem., 2006, 246, 85-99.

23. A. Abad, A. Corma and H. Garcia, Chem.-Eur. J., 2008, 14, 212-222.

24. M. A. Vannice, Catal. Today, 2007, 123, 18-22.

25. $\mathrm{N}\left(\mathrm{O}_{2}\right) / \mathrm{N}($ alcohol $)=0.3: 1$

26. After rinsing the catalyst cartridge, post-reaction, with toluene for 20 min.

27. E. Y. Lee, Y. Kim, J. S. Lee and J. Park, Eur. J. Org. Chem., 2009, 2943-2946.

28. B. H. Lipshutz and P. A. Blomgren, Org Lett, 2001, 3, 1869-1871. 